\title{
Robotic versus Open Prostatectomy: End of the Controversy
}

\author{
Michael O. Koch
}

Department of Urology, Indiana University School of Medicine, Indianapolis, Indiana

Since the early part of the last decade, the prostate cancer literature has been rife with articles purporting the utility of minimally invasive surgery for the performance of radical prostatectomy. There have been opponents citing lack of tactile feedback, an increased incidence of the need for salvage therapy and an increased complication rate, especially in the early literature comparing open and laparoscopic techniques. Some even argued that there is no difference in terms of length of hospital stay and return to normal activity, a finding which may be achievable at some centers but one which seems intuitively unlikely when broadly applied considering the findings for any other type of minimally invasive procedure. Some would also suggest that the additional costs are not justified. Conversely, the advocates would argue that the better visualization in a less bloody field has resulted in the exact opposite findings with decreased complication rates, decreased use of salvage therapy and decreased hospital stay.

Ultimately these questions can only be definitively answered with a randomized clinical trial comparing open and laparoscopic techniques, but the likelihood of successful completion of such is near nil. The predominance of robotic laparoscopic prostatectomies being performed in the United States and probably worldwide would indicate that patients have made up their minds on this issue with that being that whenever feasible, prostatectomies in the future will be performed with a robotic laparoscopic technique.

Nevertheless, demonstrations of oncologic equivalence and comparable quality of life outcomes are most important. In the absence of a randomized clinical trial, we are dependent upon single institution reports and administrative databases to gather these data with their acknowledged limitations. Single

This is the author's manuscript of the article published in final edited form as:

Koch, M. O. (2016). Robotic versus Open Prostatectomy: End of the Controversy. The Journal of Urology, 196(1), 9-10. https://doi.org/10.1016/j.juro.2016.04.047 
institution reports are basically noninformative about this issue other than what is achievable at that institution. In this issue of The Journal Pearce et al (page 76) use the National Cancer Database of the American Cancer Society and American College of Surgeons to answer some of these questions. ${ }^{1}$

Using 2 different statistical techniques to match the open prostatectomy and robotic prostatectomy groups, they found that robotic prostatectomy results in improvement in the only oncologic outcome that a surgical technique can meaningfully affect, that of positive surgical margins. Stage for stage, robotic prostatectomy was better. While these authors also found that patients treated with robotic prostatectomy needed adjuvant radiation therapy less often, this finding depends not only on complete surgical excision but also on where the surgery is performed and local practice patterns in the use of adjuvant radiation therapy.

There are certainly potential weaknesses to this study such as the potential that robotic surgery was more often performed at high volume centers compared to open prostatectomy, which might be the reason for the superior results with the robotic technique. It really doesn't matter. The conclusion that robotic prostatectomy as currently performed in the United States has superior oncologic results to open prostatectomy as it is currently being done is inescapable, despite the reason for that difference.

The issue of which approach leads to better quality of life outcomes has been inconclusive up to this point and, certainly, excellent outcomes can be achieved with either approach depending on the skill and experience of the surgeon. Studies using administrative databases based on surgical coding have suggested that incontinence is more often coded for and subsequent surgical procedures for incontinence are performed more frequently in patients undergoing robotic prostatectomy. Most of these studies are old, have multiple limitations, were conducted during the early adoption of robotic prostatectomy and have questionable validity.

O’Neil et al examined the morbidity of open and robotic prostatectomy based on validated questions in patients in the CEASAR (Comparative Effectiveness Analysis of Surgery and Radiation) and 
PCOS (Prostate Cancer Outcomes Study) clinical trials. ${ }^{2}$ The majority of the patients treated with robotic prostatectomy in this report were from the CEASAR trial and had surgery in 2011 to 2012. In this study robotic prostatectomy was associated with statistically superior urinary control at 6 months and statistically significant improvement in sexual function at both 6 months and 12 months. It is likely that in this study, as in that of Pearce et al,1 robotic prostatectomy was performed by higher volume surgeons.

Leow et al examined the morbidity of open vs robotic prostatectomy in an all-payer database, the Premier hospital database, and found that 90-day complication rates were 32\% less likely with robotic prostatectomy and transfusions were $66 \%$ less likely. ${ }^{3}$ They were also able to shed light on the issue of additional expense. These authors found that while robotic prostatectomy was associated with an additional expense of $\$ 4,528$ for the 90 -day period, the highest volume surgeons had an additional expense of only $\$ 1,990$. In their view the small incremental cost, especially in the hands of high volume surgeons, is justified by the reduction in morbidity for the patient.

From my standpoint, as someone who has substantial surgical experience with both techniques, robotic prostatectomy has superior or at least equal oncologic efficacy and complication rates compared to open prostatectomy and in 2016 any small incremental expense justifies its use. I believe the most current literature supports that view and this debate should finally be put to rest.

\section{References}

1. Pearce SM, Pariser JJ, Karrison T et al: Comparison of perioperative and early oncologic outcomes between open and robotic assisted laparoscopic prostatectomy in a contemporary population-based cohort. J Urol 2016; 196: 76.

2. O’Neil B, Koyama T, Alvarez J et al: The comparative harms of open and robotic prostatectomy in population based samples. J Urol 2016; 195: 321.

3. Leow JJ, Chang SL, Meyer CP et al: Robot-assisted versus open prostatectomy: a contemporary analysis of an all-payer discharge database. Eur Urol 2016; Epub ahead of print. 